\title{
Optimal measurement setup for damage detection in piezoelectric plates
}

\author{
G. Rus \\ Structural Mechanics $\&$ Hydraulic Engineering \\ University of Granada, Spain \\ R. Palma \\ Structural Mechanics \&6 Hydraulic Engineering \\ University of Granada, Spain \\ J. L. Pérez-Aparicio \\ Structural Mechanics \\ Polytechnical University of Valencia, Spain
}

\begin{abstract}
An optimization of the excitation-measurement configuration is proposed for the characterization of damage in PZT-4 piezoelectric plates, from a numerical point of view. To perform such an optimization, a numerical method to determine the location and extent of defects in piezoelectric plates is developed by combining the solution of an identification inverse problem, using genetic algorithms and gradient-based methods to minimize a cost functional, and using an optimized finite element code and meshing algorithm. In addition, a semianalytical estimate of the probability of detection is developed and validated, which provides a flexible criterion to optimize the experimental design. The experimental setup is optimized upon several criteria: maximizing the probability of detection against noise effects, ensuring robust search algorithm convergence and increasing the sensitivity to the presence of the defect. The measurement of voltage $\phi$ is concluded to provide the highest identifiability, combined with an excitation of the specimen by a mechanical traction transverse to the polarization direction. Sufficient accuracy is predicted for the damage location and sizing under realistic noise levels.
\end{abstract} tion.

Keywords: Piezoelectric, Finite Element Method, Inverse Problem, Probability of Detec- 


\section{Introduction}

Piezoelectric ceramics are one of the most widely used materials in all kinds of electromechanical devices due to their conversion capabilities between mechanical and electrical energies. However, the presence of damage inside the piezoelectric plates degrade the functionality they were designed for. For this reason, many researchers study the behaviour of these ceramics with the presence of defects, either from an analytic viewpoint ([28], [29] and [14]), numerical ([20], [3] and [11]) or experimental ([19] and [9]), in order to find a fracture criteria to predict failure.

In recent years, identification Inverse Problems (IP) have been applied in many studies (see [12], [15], [17], [24] and [31]). Examples of identification IP for elasticity are [32] and [4]. However, due to the intrinsic coupling of magnitudes, the formulation of the piezoelectric problems is more complex than in elastic problems. Despite this difficulty, the electric field and its coupling with the elastic field should be exploited in monitoring piezoelectrics and in the solution of the IP. The IP techniques have been applied to find the elastic, dielectric and coupling properties of piezoelectric ceramics. In [10] a Cost Functional (CF) was defined as the difference between electric impedances observed in laboratory and those obtained after solving the direct problem by the Finite Element Method (FEM). A similar CF was used in [22] and [23], which was minimized using Genetic Algorithms (GA). In this sense, GA was applied in [5] and [13] to solve the IP in elastic structures. Based on crystallographic criteria, in [26] was formulated the CF as the difference in the orientation distribution function (which provides a statistical description of the orientation). In [2] and [1] was proposed an IP to obtain the constitutive properties of composite plate specimens with surface bonded piezoelectric patches or layers, where the CF was the difference between the experimental and FEM-predicted eigen-frequencies, and its minimization was carried out using two strategies: a gradient-based method, and neural networks. Finally, in [35], an IP was solved to determine the non-uniform polarization of the piezoelectric ceramics.

After revising the existing literature, three questions appear to remain open: i) the use of the IP to detect damage, ii) once the damage identification IP is formulated, which is the optimal experimental measurement setup for improving the sensitivity, and iii) what is the Probability of Detection (POD) in this optimal configuration. An IP for locating and sizing defects in a PZT-4 ceramic (see properties in [20]) under plane strain is presented here. In this framework, the forward problem is solved by a FEM formulated in [20] and implemented in the research code FEAP (see [33]). In addition, the solution in a domain with a small circularly shaped defect is accurately solved by incorporating a parametrized and optimized finite element mesh, which combines a good precision with a low CPU time. Once the numerical problem is solved, a standard least-squares $\mathrm{CF}$ is defined to measure the discrepancy between experimental and FEM-predicted measurements. Since no experimental measurements are available for this work, those are simulated numerically by adding experimental noise (characterized by a gaussian distribution) to results obtained by FEM. Afterwards, the CF is minimized using GA and a gradient-based method, in order to capture local minima. Nevertheless, the main goal of this work is to determine which excitation and measurement magnitudes provide the optimal configuration in terms of high sensitivity to the defect and low sensitivity to noise and model uncertainties (therefore, sophisticate damage models and inverse problem techniques have not been developed). Finally, in order to obtain the optimal configuration, a number of excitation-measurement combinations are studied, and an estimation of the POD is developed from the formulation given in [25], which has previously been validated using Monte Carlo (MC) techniques (see [27] and [18]). 


\section{Piezoelectric governing equation}

Piezoelectric materials have the ability to generate an electric charge in response to applied mechanical stress and vice versa. The continuum governing equations of the mechanical and the electrical behaviour are combined by some coupling terms, which are well defined for the linear case (which is assumed in this work). A plane strain geometry is assumed, in which all field variables depend on $(x, z)$, where $z$ is the polarization $\mathbf{P}$ direction of material. The piezoelectric constitutive equations are given by [28] as,

$$
\mathbf{S}=\overline{\mathbf{s}}^{D} \mathbf{T}+\overline{\mathbf{g}}^{t} \mathbf{D} \quad ; \quad \mathbf{E}=-\overline{\mathbf{g}} \mathbf{T}+\overline{\boldsymbol{\beta}}^{T} \mathbf{D}
$$

where $\mathbf{S}, \mathbf{T}, \mathbf{E}, \mathbf{D}, \overline{\mathbf{s}}^{D}, \overline{\mathbf{g}}$ y $\overline{\boldsymbol{\beta}}^{T}$ denote deformation, stress, electric field and displacement, and elastic, coupling and dielectric properties measures to constant stress $(.)^{T}$, in reduced or effective form, respectively. In absence of body forces and electric charge density, the piezoelectric behaviour is modelled by Gauss law, the mechanical equilibrium equation, the equations that relate the electric and voltage fields, and the compatibility equations,

$$
\begin{gathered}
\nabla \cdot \mathbf{D}=0 \quad ; \quad \mathbf{E}=-\nabla \phi \\
\nabla^{s} \cdot \mathbf{T}=0 \quad ; \quad \mathbf{S}=\frac{1}{2}\left(\nabla \mathbf{u}+\nabla \mathbf{u}^{t}\right)
\end{gathered}
$$

where $\mathbf{u}=(u, w)$ denotes the displacement in directions $x$ and $z$, respectively, and $\phi$ is the electric potential or voltage. Finally, the following standard sign criteria is used: the electric field and stress values are considered positive for the direction of polarization of the material and for tractions, respectively.

\section{Forward problem}

The proposed problem is the characterization of damage inside the piezoelectric material. This problem can be stated as the calculation of some location and size parameters of the defect by means of an Inverse Problem (IP). The IP can be defined as a counterpart of the forward problem as follows: if the direct problem consists of finding the response of a known system (which can be done by a numerical model), the inverse problem consists of finding an unknown part of the system (in our case, damage characterization) given a known part of the response (in our case, measurements on an accessible part of the monitored specimen). The next section describes how the IP requires an iterative evaluation of the forward problem, whose solution is described in the following.

\section{$3.1 \quad$ FEM}

The numerical tool selected for solving the response of the model (forward problem) is the Finite Element Method (FEM). A 9-node quadratic finite element, that solves the model given by the constitutive equations described in (1), and the equilibrium and compatibility equations (2), has been implemented. This element was developed in [20], using the research academic finite element code FEAP [33], and was validated against the analytical solutions obtained by [28] for an elliptical hole inside an infinite plane strain plate under electromechanical loads. 


\subsection{Specimen description}

The system in which the defect will be sought is defined by the geometry, material of the specimen, the boundary conditions, and applied loads and the measuring points as output data.

The specimen consists of a ceramic plate with a defect defined by a loss of effective material. The PZT-4 plate is considered as square shaped of size $L_{x}=L_{z}=6[\mathrm{~cm}]$, as shown in Figure 1. This sample is excited by electrical or mechanical loads, and its response is measured at $N_{i}=25$ points along the lower boundary of the plate. The selection of electrical or mechanical load is one of the goals of this study. It should be noted that the piezoelectric coupling makes mechanical load generate voltages (direct effect) and the electrical loads generate mechanical displacements (inverse effect).

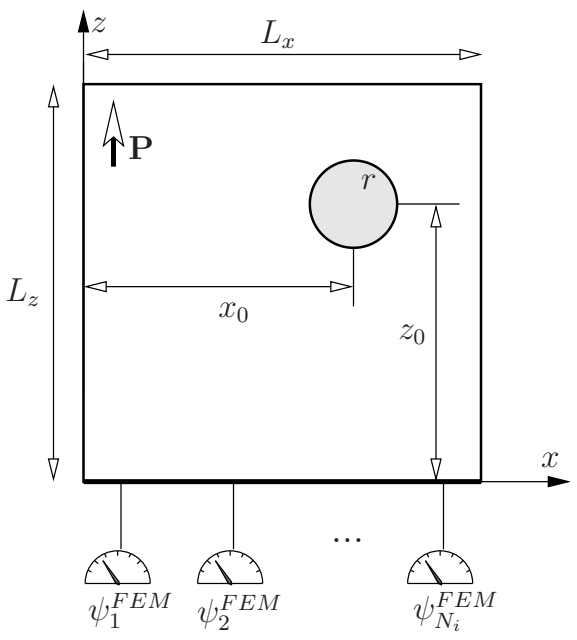

Figure 1: Experimental setup for detecting the defect.

The boundary conditions are selected to avoid rigid solid motions, and including the prescribed excitation loads for the measurement as depicted in Figure 11.

The measurements generated by a forward problem FEM are a vector of values at every measuring point denoted by $\psi^{F E M}=\left(u^{F E M}, w^{F E M}, \phi^{F E M}\right)$, whereas the experimental measurements are denoted by $\psi^{E X P}$. Since no experimental measurements are available in this study, they are simulated by $\psi^{F E M}$ as,

$$
\psi_{i}^{E X P}=\psi_{i}^{F E M}+\xi_{i} R M S\left(\psi_{i}^{F E M}\right) \sigma
$$

where $i=1, \ldots, N_{i}, \xi_{i}$ are random variables generated by a gaussian distribution of mean 0 and standar deviation 1: $\xi_{i}=N(0,1), \sigma$ is a parameter defined to study the influence of the noise on the final detection and $R M S\left(\psi_{i}^{F E M}\right)$ is the root mean square given by:

$$
R M S\left(\psi_{i}^{F E M}\right)=\sqrt{\frac{1}{N_{i}} \sum_{i=1}^{N_{i}}\left(\psi_{i}^{F E M}\right)^{2}}
$$

\subsection{Damage description}

The damage is defined as a loss of effective material, assumed to be represented by a circularly shaped cavity of radius $r$ (and area $A=\pi r^{2}$ ), described in Figure 1. Such a simplified damage 
model is assumed to suffice for the purpose of optimize the excitation-measurement configuration.

The boundary conditions in the isolated cavity are considered as impermeable (see [16] for a careful discussion of the selection of the boundary conditions of cracks and defects). If exact boundary conditions were considered, the interior of the defect would need a mesh, as done in [20], which increases the complexity and number of elements and hence the computational cost. Since this study is focused on the location and extent of a circular defect, the impermeable approximation provides acceptable results, as [28], [29] and [20] proved.

\subsection{Mesh generation}

The finite element mesh on the geometry, consisting of a cavity inside a square, has some requirements related to the stability during the iterative geometry variation (i.e. a smooth variation of the geometry should always produce a smooth variation on the remeshing) in combination with accuracy and speed demands (since the gradient-based search algorithm and especially the GA require a large number of forward problem evaluations). An algorithm that automatically generates a high quality finite element mesh has been implemented and is described in this section.

Given a square domain with a random $\left(x_{0}, y_{0}, r\right)$ circle hole inside (see Figure 1), a multi-block structured mesh has to be generated automatically, which guarantees the first stability condition. For the accuracy demand, the meshes should fulfill two conditions:

1. Due to the electrical and stress concentrations around the damage, (see [20]), the elements should to be smaller near the hole.

2. The maximum element size should be selected to provide an error in the measurements below an acceptable threshold.

A fully automatic algorithm to construct multi-block structured 2D meshes is developed and used. This algorithm consists of three steps:

Step 1: To subdivide the domain into simple blocks. The domain is subdivided by means of Medial Axis Transform (MAT), see [30]. Given a two-dimensional domain, its MAT is the locus of centres of maximal empty circles inside the domain. This technique allows to obtain the skeleton of the domain. Figures 2 (a) and (b) show the domain (thick line), the skeleton obtained through MAT (semi-dotted line) and the connection lines between intersection point $P_{i}^{2}$ and $P_{i}^{1}$ (dotted line), $i=1,2,3$.

Step 2: To mesh each block. Quadrilateral elements inside each block, described by four bounding curves, are generated using Transfinite Interpolation (TFI), see [34]. TFI is a technique to draw meshes mapping the unit square (computational domain: $0<\xi<1,0<\eta<1$ ) onto the interior of the physical domain $(x-z)$.

Step 3: To concentrate elements. To concentrate elements close to the circular hole, a Stretching Function (SF) is used, see [34]. A SF is a monotonously increasing function defined in the interval $(0,1)$ in the computational domain. A SF of the hyperbolic tangent type was used with a stretching parameter $\omega=2.5$. Figures 4 (a) and (b) show the meshes of the previous Step (see Figure 3) when $\mathrm{SF}$ is applied. Now, the nodes are redistributed and progressive meshes are obtained. 


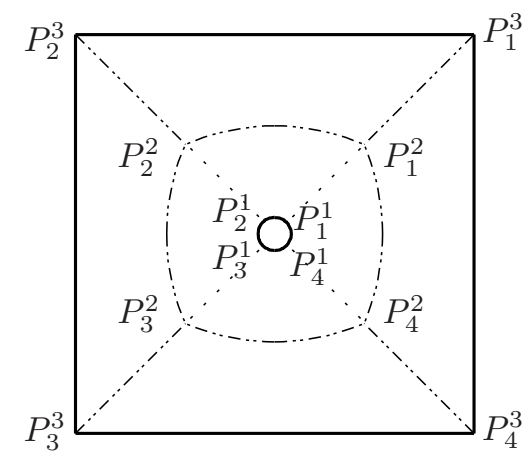

(a)

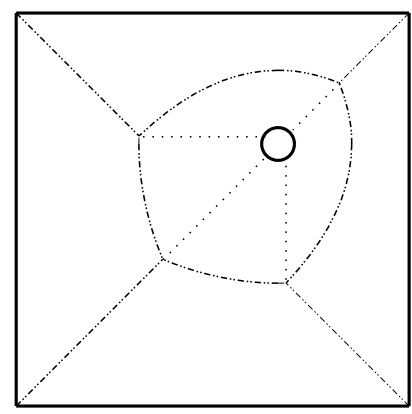

(b)

Figure 2: Step 1. Block subdivision created by MAT for: (a) a hole in the centre of the plate, (b) a hole in a random position.

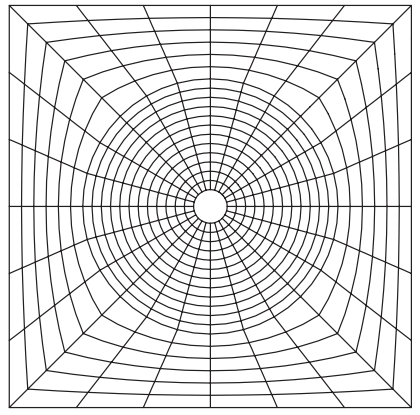

(a)

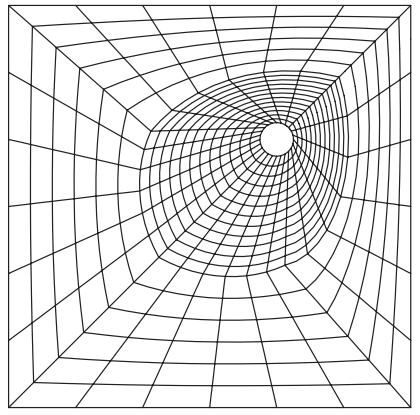

(b)

Figure 3: Step 2. Multi-block structured meshes for: (a) a hole in the centre of the plate, (b) a hole in a random position.

The element size was determined by a convergence study, in which the error on measurement point and the consumed CPU time were monitored. The error was defined by the parameter $\eta$,

$$
\eta=\left|\frac{\phi^{E X A}\left(x_{0}, z_{0}, r\right)-\phi^{F E M}\left(x_{0}, z_{0}, r\right)}{\phi^{E X A}\left(x_{0}, z_{0}, r\right)}\right| \times 100
$$

where $\phi^{E X A}\left(x_{0}, z_{0}, r\right)$ and $\phi^{F E M}\left(x_{0}, z_{0}, r\right)$ are the exact and FEM-computed electric potentials at the edge of the circular cavity (where the field will show the maximum concentration, and the maximum error will be located). Since there is no available analytical solution for the electric potential on the edge of a finite plate, the exact solution is estimated by a highly refined FEM mesh (using 9600 elements).

Figure 5 shows $\eta$ versus the total number of elements. It is shown that the threshold of $10^{-3} \%$ error is reached before 1000 elements. The error correctly keeps decreasing after that point. In this work, a mesh consisting of 1176 elements is used, where $\eta=4.8 \times 10^{-4} \%$ and the CPU time of 6 [s] with a PC of $1[\mathrm{~Gb}]$ of RAM memory and Linux operating system. 


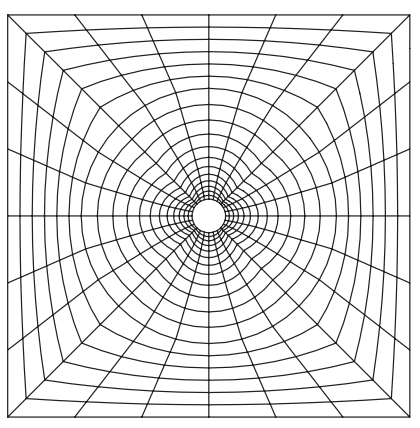

(a)

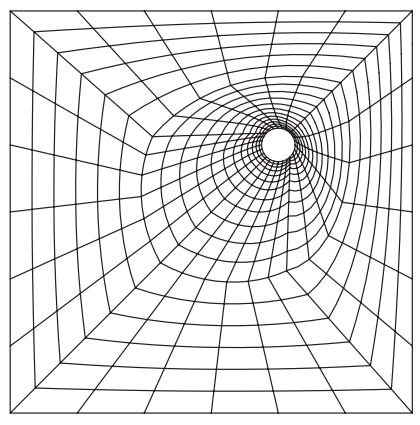

(b)

Figure 4: Step 3. Progressive multi-block structured meshes for: (a) a hole in the centre of the plate, (b) a hole in a random position.

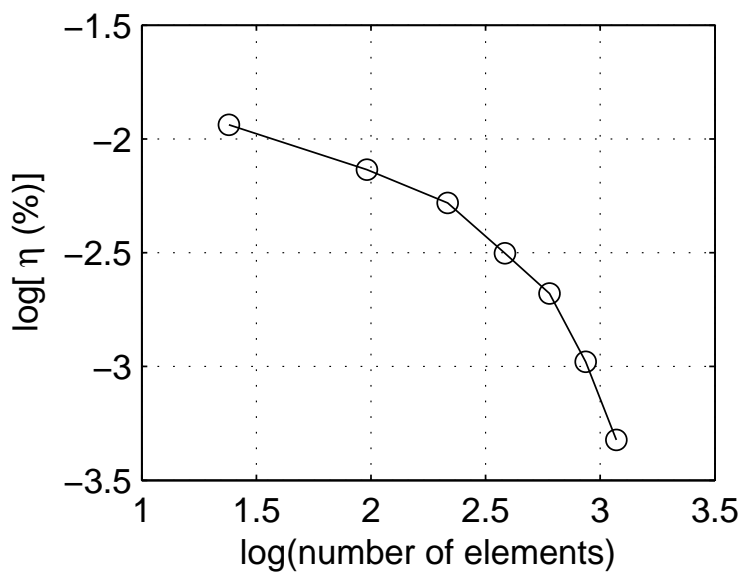

Figure 5: FEM error estimation when increasing the number of elements.

\section{Inverse problem}

The characterization of the defect, defined as the calculation of its location and size parameters, is the goal of this formulation. This characterization is performed in three steps: i) the specimen is excited by mechanical or electric loads, ii) a measurement of the response of the specimen (displacements or voltages) is recorded, and iii) the recorded information is interpreted by the IP algorithm to obtain the parameters of the defect. The IP is stated as characterizing the defect, which is a part of the model, given the known response. The solution of the IP is proposed by setting up the cost functional, the parametrization and the minimization problem.

\subsection{Cost functional}

The CF, often times referred to as fitness function or objective function, $f$, is defined as a quadratic difference between the experimental and FEM-predicted measurements,

$$
f=\frac{1}{2 N_{i}} \sum_{i=1}^{N_{i}}\left(\psi_{i}^{E X P}-\psi_{i}^{F E M}\right)^{2}
$$


where $N_{i}=25$ is the number of measuring points.

In contrast to gradient-based algorithms, for which the $\mathrm{CF}$ is defined as $f$, when the minimization is carried out by genetic algorithms, or other heuristic algorithms, the CF is usually defined in an alternative way $f^{L}$,

$$
f^{L}=\log (f+\varepsilon)
$$

where $\varepsilon$ is a small adimensional value (here adopted as $\varepsilon=10^{-16}$ ) that ensures the existence of $f^{L}$ when $f$ tends to zero. In addition, as argued in [7], this new definition of the CF usually increases the convergence speed of the minimization algorithms.

\subsection{Parametrization}

In the context of inverse problems, parametrization of the model means to characterize the sought solution (the defect in this case) by a set of parameters, which are the working variables and the output of the inverse problem. The choice of parametrization is not obvious, and it is a critical step in the problem setup, since the inverse problem is a badly conditioned one, in the sense that the solution may not be stable, exist or be unique, and the assumptions on the damage model that allow to represent it by a set of parameters can be understood as a strong regularization technique. In particular, a reduced set of parameters is chosen to facilitate the convergence of the search algorithm, and they are also defined to avoid coupling between them.

The damage location and size estimation problem presented suggests the definition of the immediate parameters $x_{0}, z_{0}$ to characterize the location of the center of the defect, and $r$ to define the radius of that circle that represents the extent of the defect (see Figure 1). The parameters obtained as the estimated solution of the IP are grouped in a vector $\mathbf{p}=\left\{p_{i}\right\}=\left\{x_{0}, z_{0}, r\right\}$, whereas the parameters that represent the true characteristics of the defect are denoted by $\tilde{\mathbf{p}}$.

One should bear in mind that there is a strong relationship between the number of input and output data (number of measurements and number of output parameters), which is also responsible for the conditioning of the problem. In particular, the number of measurements must be equal or larger (preferably) than the number of parameters.

\subsection{Minimization}

The IP of defect evaluation can be stated as a minimization problem, that may be constrained, as finding $p_{i}$ such that,

$$
\min _{p_{i}} f(\mathbf{p})
$$

The GA is a heuristic optimization technique based on the rules of natural selection and genetics (see [8]). It simulates the mechanism of survival competitions: the superiors survive while the inferiors are eliminated. The GA has been applied in different research topics, due to its simple implementation procedure (see Figure 6).

A population of individuals (called chromosomes) is randomly generated. The population comprises a group of chromosomes to represent possible solutions in a problem domain. Each solution is generated by computing the cost functional, for which one forward problem is solved independently, as depicted in Figure 7. Genetic operators such as crossover and mutation are applied to obtain other population. Then, the child chromosomes with higher fitness replace some of their 


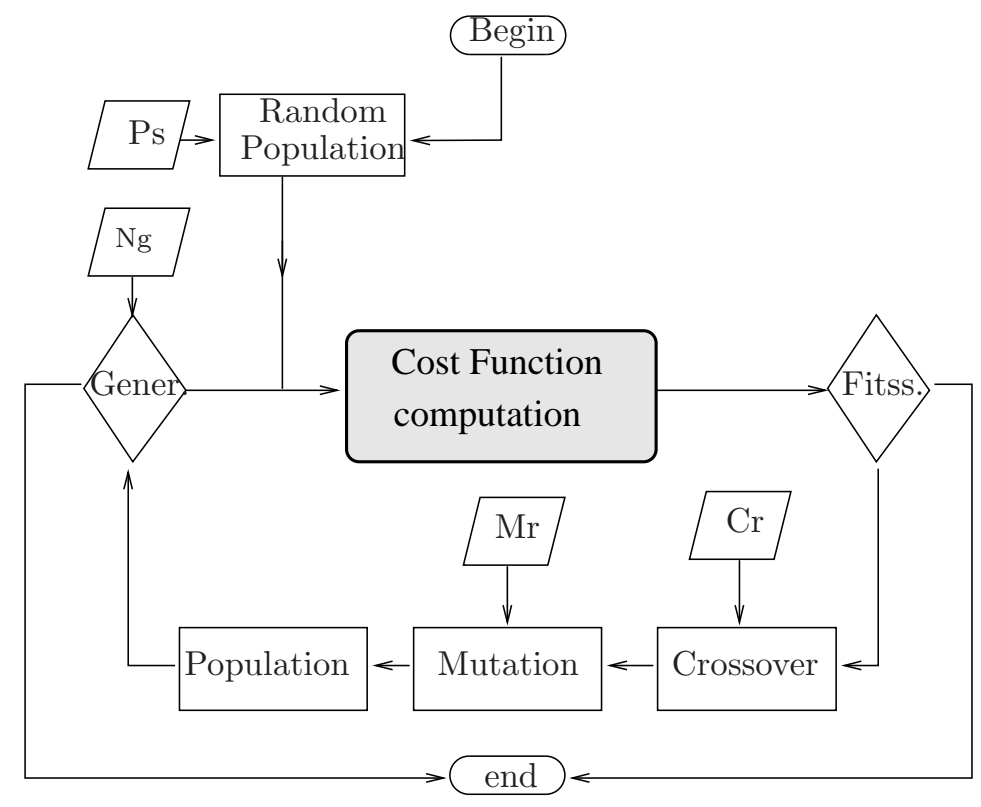

Figure 6: Flow chart of the inverse problem solution by Genetic Algorithms. Gener., Fitss., Mr, Cr, Ps and Ng stand for generations, fitness or cost function, mutation ratio, crossover ratio, population size and number of generations, respectively.

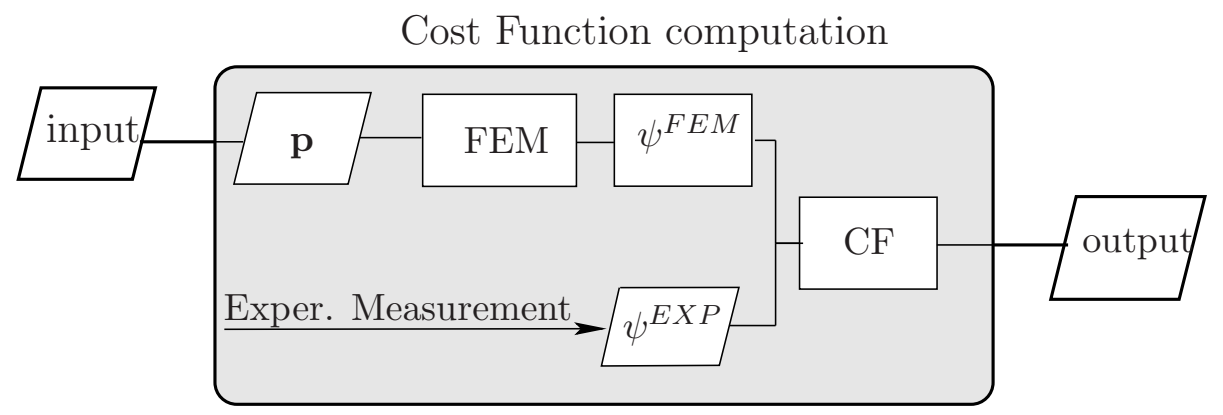

Figure 7: Flow chart of the computation of the cost functional from the forward problem. The forward problem is solved by the FEM. 
parent chromosomes. The process runs until a stopping criterion (like the number of generations) is reached.

The BFGS (Broyden, Fletcher, Goldfarb, and Shanno) is an effective and robust gradient-based algorithm that use the quasi-Newton methods. These methods build up curvature information at each iteration to formulate a quadratic model problem (Hessian update), see [6].

\section{Probability of detection}

The economy of the health monitoring industry is based on several interrelated concepts, but the basic one is the Probability of Detection (POD). The POD gives an idea of the probability that a defect is positively detected, given a specimen, a defect size and some noise and system uncertainty conditions. The aim of the following section is to provide an estimation of this probability as a function of such variables.

The detection and characterization of defects is based on the interpretation of the alterations of the measurements due to the presence of the defect. Other model uncertainties and system noises also alter these measurements. We can estimate the POD by the probability that the alteration of the measurement caused by the defect is larger than that caused by the noise. If we label the alteration on the measurement readings caused by the defect as the SIGNAL component, and the alteration generated by the noise as NOISE, the former definition can be formulated as (see [25]),

$$
P O D=P\left(\frac{|\mathrm{SIGNAL}|^{2}}{|\mathrm{NOISE}|^{2}}>1\right)
$$

\subsection{Signal and noise components}

Figure 8 shows that the recorded measurement $\psi^{F E M}=\left(u^{F E M}, w^{F E M}, \phi^{F E M}\right)$ depends linearly on the area $A$ of the defect (assuming circular shape of radius $r$ ), where the defect is considered to be centered at the plate (parameters $x_{0}=z_{0}=3[\mathrm{~cm}]$ ) and the applied load is transversal to the polarizaction direction $T_{x x}^{a p}=1[\mathrm{kPa}]$. In the case of displacement measurements $u^{F E M}$ and $w^{F E M}$ this linearity is only valid for the range $A \in(0,1.5)\left[\mathrm{m}^{2}\right]$. Furthermore, in the case of voltage measurements $\phi^{F E M}$, nonlinear behaviour is observed on the numerical results for small defects, $A \in(0,0.015)\left[\mathrm{m}^{2}\right]$, because the FEM is unable to correctly capture the small perturbation (the mesh would need better refinement).

From the definition of the simulated noise in (3), the dependency of the variation of the measurement with increasing noise is also linear. These two considerations about linearity support the proposal that the measurements on a specimen with noise and with defect can be expressed as Taylor series expansion centered at the case without noise and without defect, and neglecting higher order terms (hot) than linear,

$$
\psi_{i}(A, \sigma)=\psi_{i}(0,0)+\underbrace{A \frac{\partial \psi_{i}}{\partial A}(0,0)}_{\text {SIGNAL }}+\underbrace{\sigma \frac{\partial \psi_{i}}{\partial \sigma}(0,0)}_{\text {NOISE }}+\text { hot }
$$

where $i=1, \ldots, N_{i}$ are the measuring points (see Figure 1). The first term on the right hand side is the measurement at point $i$ without noise nor defect. The second term is the alteration of that 

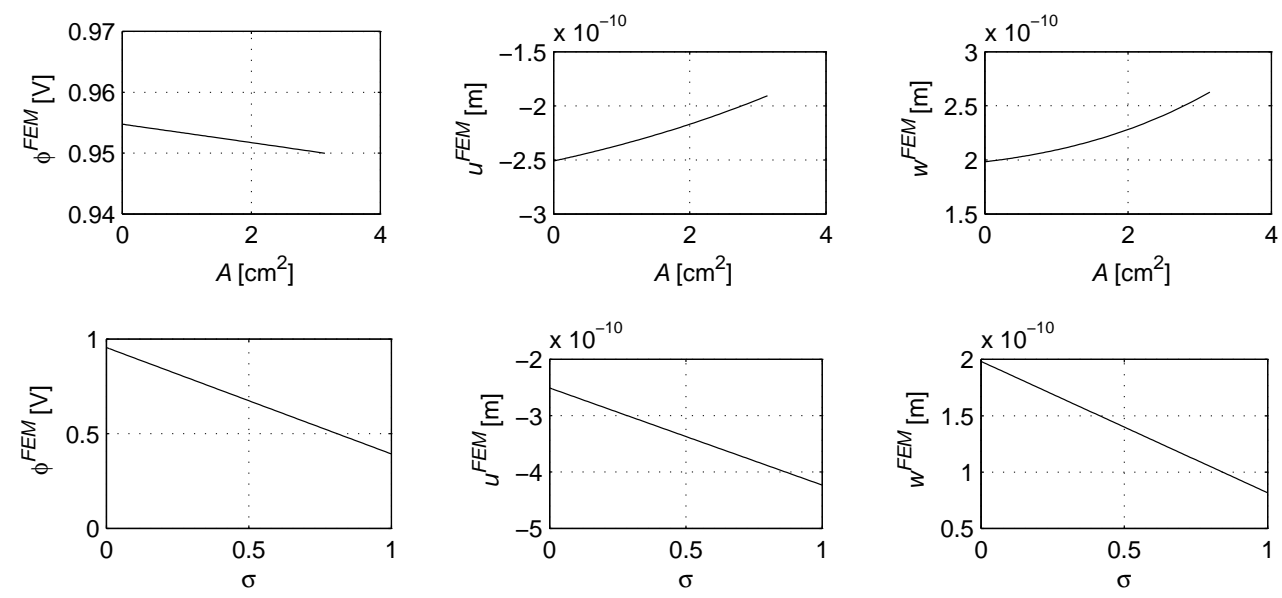

Figure 8: Linear dependency of measurements upon size of defect. Measurements at the center point for an increasingly large defect, starting from the case with no defect.

measurement due to the presence of the defect only, and is labelled SIGNAL, following the reasoning above. The third term is the alteration of the signal originated by the noise only (NOISE).

\subsection{Finite differences}

The second and third terms of the Taylor series in (10) depend on the sensitivity of the measurements on the area and the noise respectively, and can therefore be computed by finite differences,

$$
\begin{aligned}
& \frac{\partial \psi_{i}}{\partial A}\left(A_{0}, 0\right)=\psi_{i, A}\left(A_{0}, 0\right)=\frac{\psi_{i}\left(A_{0}+\Delta A, 0\right)-\psi_{i}\left(A_{0}-\Delta A, 0\right)}{2 \Delta A} \\
& \frac{\partial \psi_{i}}{\partial \sigma}(0,0)=\psi_{i, \sigma}(0,0)=\frac{\psi_{i}(0, \Delta \sigma)-\psi_{i}(0,0)}{\Delta \sigma}
\end{aligned}
$$

where $A_{0} \rightarrow 0$ is a small defect used to guarantee that the FEM captures the perturbations produced at small $\Delta A$ (since the case $A=0$ with no defect needs to be computed with a topologically different mesh), in order to compute $\psi_{i_{A}}\left(A_{0}, 0\right) \approx \psi_{i, A}(0,0)$. In addition, a central difference scheme, which yields an error of the order $O\left(\Delta A^{2}\right)$, becomes available. Since the noise component is linear by definition, a forward difference scheme is adopted, whose $O(\Delta \sigma)$ error is sufficient.

Some authors [27] propose that the parameters $\Delta A$ and $\Delta \sigma$ should be two orders of magnitude smaller than the values at which the derivative should be computed. However, an estimation of these parameters is studied in Figure 9. It shows $\psi_{i, A}(0,0)$ and $\psi_{i, \sigma}(0,0)$ versus $\Delta A$ and $\Delta \sigma$, respectively, for a defect at the center of the plate. $\Delta A=\Delta \sigma=10^{-1}$ is shown to produce a stable value of the derivative for the case of the single measurement represented, but the same result is obtained for all 25 measuring points.

On the other hand, (3) yields by direct derivation the last term of the Taylor expansion,

$$
\frac{\partial \psi_{i}}{\partial \sigma}=\xi_{i} R M S\left(\psi_{i}^{F E M}\right)=\xi_{i} R M S
$$



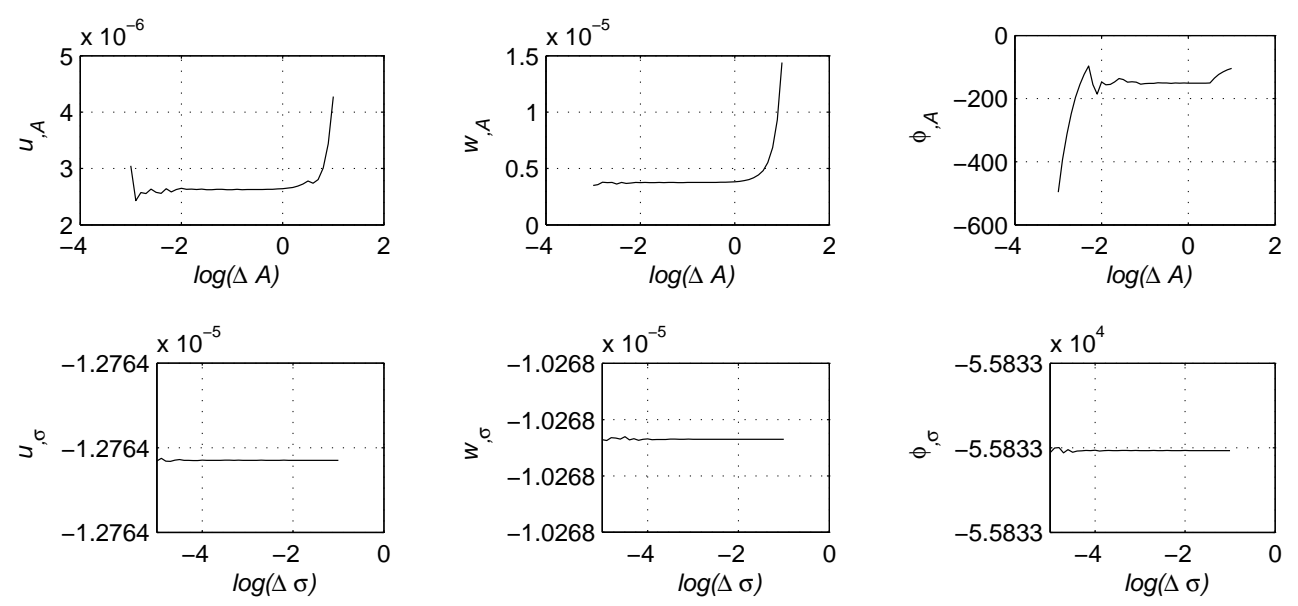

Figure 9: Estimation of the finite difference parameters $\Delta A$ and $\Delta \sigma$. The range where the derivative of $u, w$ or $\phi$ presents a stable value provides a valid range of finite difference parameter.

\subsection{Analytical estimation of the POD}

Equations (10), (12) and the relationship $\left|Y_{i}\right|^{2}=\frac{1}{m} \sum_{i=1}^{m} Y_{i}^{2}$, can be combined into (9) to obtain,

$$
\mathrm{POD}=\mathrm{P}\left(\frac{A^{2} \frac{1}{N_{i}} \sum_{i=1}^{N_{i}}\left(\psi_{i, A}(0,0)\right)^{2}}{\sigma^{2} R M S^{2} \frac{1}{N_{i}} \sum_{i=1}^{N_{i}} \xi_{i}^{2}}>1\right)=\mathrm{P}\left(A^{2}>\frac{R M S^{2} \sigma^{2} \sum_{i=1}^{N_{i}} \xi_{i}^{2}}{\sum_{S_{i}}^{\sum_{i=1}\left(\psi_{i, A}(0,0)\right)^{2}}}\right)
$$

If the noise generator $\xi_{i}$ is a random variable, the POD is a probability of the stochastic variable $A^{2}$ (square of the area of the defect), described by the cumulative probability density function $F$,

$$
P O D=F\left(\frac{R M S^{2} \sigma^{2} \sum_{i=1}^{N_{i}} \xi_{i}^{2}}{S_{A}}\right)
$$

Using Monte Carlo techniques and error propagation theory the noise in the measurement points can be concluded to follow a normal distribution (see [18]). Assuming this distribution, the squared sum of the noise $\xi_{i}$ is known to follow a Chi-square distribution, since $\sum_{i=1}^{N_{i}} \xi_{i}^{2} \longrightarrow \chi_{N_{i}}^{2}$ (e.g. [21]). The parameter of the Chi-square distribution is the number of degrees of freedom $N_{i}$, which in this case is the number of measurement points. In the case that $N_{i}>10$, the Chi-square distribution can be approximated by a Gaussian or normal $N$ distribution $\chi^{2}\left(N_{i}\right) \approx N\left(N_{i}-2 / 3, \sqrt{2 N_{i}}\right)$ with mean $N_{i}-2 / 3$ and standard deviation $\sqrt{2 N_{i}}$. This approximation in (14) yields,

$$
A^{2} \longrightarrow N\left[\frac{R M S^{2} \sigma^{2}\left(N_{i}-2 / 3\right)}{S_{A}}, \frac{R M S^{2} \sigma^{2} \sqrt{2 N_{i}}}{S_{A}}\right]
$$

Since $F(x)=\int_{-\infty}^{x} f(y) d y$ is the cumulative of the normal probability density function $f$, whose inverse is $x=G(F(x))$, the useful defect area to noise ratio $A / \sigma$ can be expressed from (15) given 
a POD level as,

$$
\frac{A}{\sigma}=\sqrt{\frac{R M S^{2}\left(N_{i}-2 / 3\right)}{S_{A}}\left(1+G[P O D] \frac{\sqrt{2 N_{i}}}{N_{i}-2 / 3}\right)}
$$

Note that the analytical expression (15) is only valid for noise with normal distribution at the measurement points. In addition, note that, since $S_{A}$ is dependent on the damage location, different values of the POD appear for different positions of the damage. A pessimistic criterion is adopted in this work, in which a grid of possible damage locations are evaluated, and the lowest POD is selected, which corresponds to the most difficult damage to detect.

\subsection{Validation of the POD}

Three Monte Carlo (MC) simulations (see [27] and [18]) are carried out to validate and calculated the robustness of the POD's formulation obtained. Each simulation is performed evaluating 1000 times expression (14) for a noise level of $\sigma=2 \%$ and $R M S=95.5[\mathrm{kV}]$ where $\xi_{i}$ is considered as the random variable of zero mean and unity standard deviation, according to its definition. In order to verify the robustness of the POD estimate against deviations of the distribution from normality, three samples of $\xi_{i}$ are generated, with (i) a normal random sample, (ii) a sample containing the measurements that don't fulfill the Kolmogorov-Smirnov normality test with $5 \%$ significance level (i.e. the $5 \%$ of the measurements that are furthest away from the normal distribution), and (iii) a sample outside the normality test with significance level $0.5 \%$.

The results are presented in Figure 10, where the POD is presented for $\psi=\phi$, as given by Equation (15) and also using the three Monte Carlo simulations described before. Analytical and MC, using the sample (i), curves match well, which allows to conclude the correctness of the analytical estimation of the POD. On the other hand, MC using samples (ii) and (iii), with distributions away from normality, present a deviation compatible with the lack of normality, but still fall within an admissible band, showing that the formulation is sufficiently robust when the measurements are not normally distributed.

\section{$6 \quad$ Numerical results}

The goal of the numerical results is, first, to validate the applicability of the semianalytical estimate of the POD developed for piezoelectric materials, and second, to obtain conclusions about which experimental design or measurement setup performs better in characterizing damage on piezoelectrics. To the latter end, three independent criteria are evaluated numerically. The effects of the measurement/loading combination on (i) the measurements, (ii) the CF and (iii) the POD are studied for a set of benchmark configurations (i.e. specimens and the measuring magnitude and location). Criteria (i) and (ii) are qualitative, whereas (iii) is quantitative. The goal is to extract some a priori thumb rules that allow to select those with a more accessible minimum in the CF (solution of the IP), and guarantee satisfactory results for a minimization algorithm such as the GA or BFGS, since the large number of FEM simulations it calls, makes it a computationally expensive algorithm. 


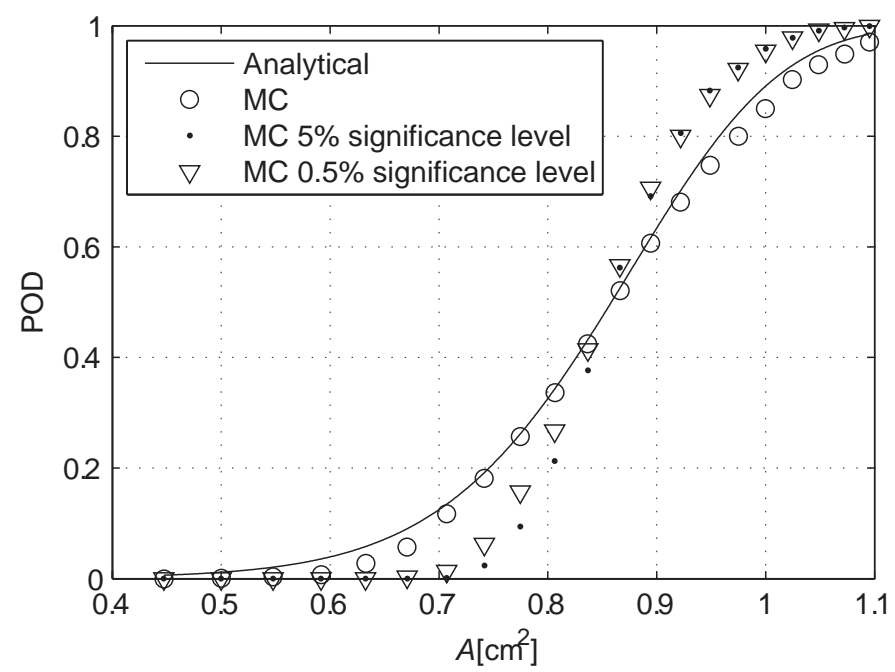

Figure 10: Comparison between analytical expression of the POD and Monte Carlo simulations of the POD, for differents significance levels and noise of $\sigma=2 \%$.

\subsection{Specimen configurations}

Consider an experimental configuration given by a PZT-4 plate, polarized in the $z$ direction, of dimensions $L_{x}=L_{z}=6[\mathrm{~cm}]$. All the measurements are taken at the lower boundary of the plate, at equally spaced sensors noted as $\psi_{i}^{M E F}$ in Figure 1.

Table 1 shows the labelling of all the excitation-measurement combinations, where $T_{x x}^{a p}=1$ $[\mathrm{kPa}], T_{z z}^{a p}=1[\mathrm{kPa}], E_{x}^{a p}=1[\mathrm{MV} / \mathrm{m}]$ y $E_{z}^{a p}=1[\mathrm{MV} / \mathrm{m}]$ are the stress or electric fields applied in direction $x$ or $z$. Figure 11 shows the boundary conditions and the procedure to simulate loads by FEM for each configuration. Note that the electric potential is always set to zero along the top boundary of the plate, except for the configuration XII where the potential is zeroed at the lower boundary, since it requires a reference point.

\begin{tabular}{||c|c|c|c||}
\hline Load & $u$ & $w$ & $\phi$ \\
\hline \hline$T_{x x}^{a p}$ & $I$ & $I I$ & $I I I$ \\
\hline$T_{z z}^{a p}$ & $I V$ & $V$ & $V I$ \\
\hline$E_{x}^{a p}$ & $V I I$ & $V I I I$ & $I X$ \\
\hline$E_{z}^{a p}$ & $X$ & $X I$ & $X I I$ \\
\hline
\end{tabular}

Table 1: Labelling of all load-measurement combinations.

\subsection{Effects on measurements}

The first way to evaluate and compare the results of every configuration is to observe the effects of the combination on the measurements, which gives an idea of the sensitivity of these. Despite the subjectiveness of their interpretation, the configurations that show little or no effect due to the presence defect may be directly discarded. 


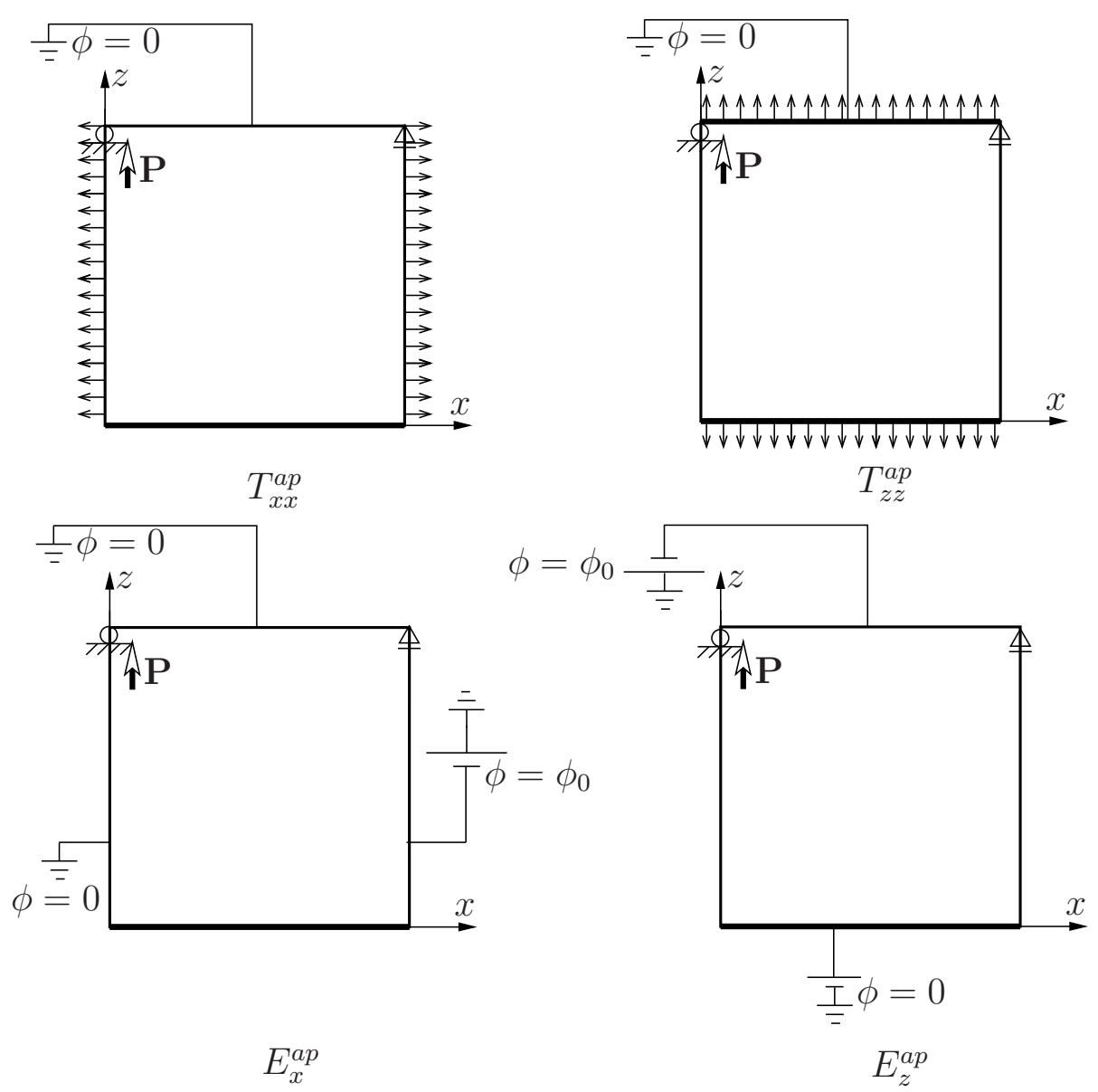

Figure 11: Boundary conditions and loading in the FEM simulation. $\phi_{0}=-0.06[\mathrm{kV}]$.

Figure 12 shows the measurements, obtained by the FEM, for all the configurations for the cases of no defect (undamaged) and with a defect located at $x_{0}=3.5[\mathrm{~cm}]$ and $z_{0}=2[\mathrm{~cm}]$ with increasing radii $r=0.5,1[\mathrm{~cm}]$. In all cases, the presence of the defect alter the measurements, excepting for configuration $X I I$. The reason is that applying $E_{z}^{a p}$ requires the voltage to be fixed at the top and bottom boundaries of the plate, which prescribes as zero the potential at the measuring points. In addition, configurations $I I, I I I, I V, V, V I, V I I I, X I$ provide measurements with a larger sensitivity to the presence of the defect. Therefore, the best configurations are:

$$
C_{1}=\{I I, I I I, I V, V, V I, V I I I, X I\}
$$

\subsection{Effects on cost functional}

The shape of the CF provides another subjective way to evaluate the measurement sensitivities. The qualitative criteria to evaluate them are the following,

- CF functions with only global minima are considered to be better. When local minima exist, the search algorithm performs computationally more expensively, and a risk for a wrong solution exists. 


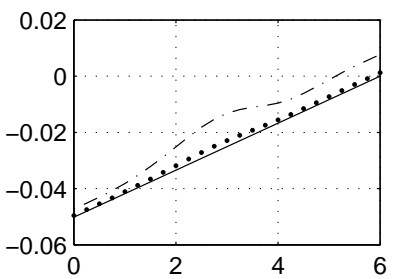

(a) $I$

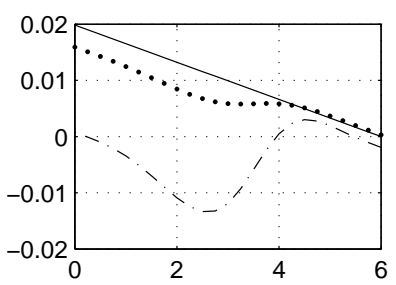

(d) $I V$

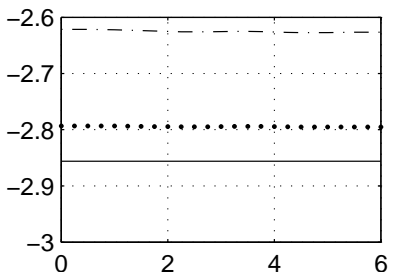

(g) $V I I$

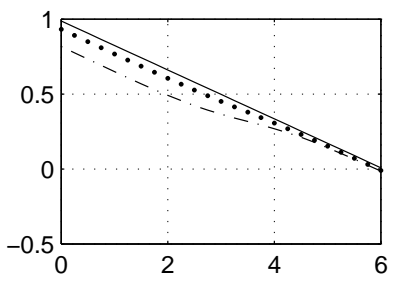

(j) $X$

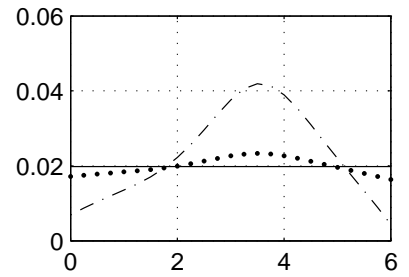

(b) $I I$

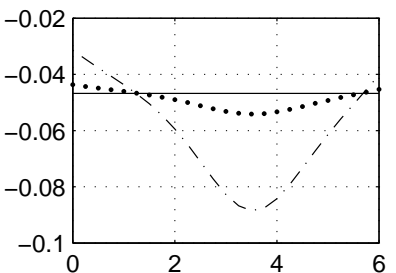

(e) $\mathrm{V}$

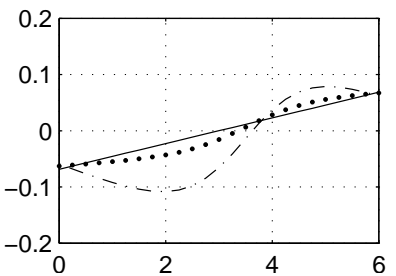

(h) VIII

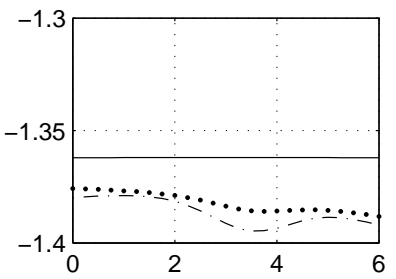

(k) $X I$

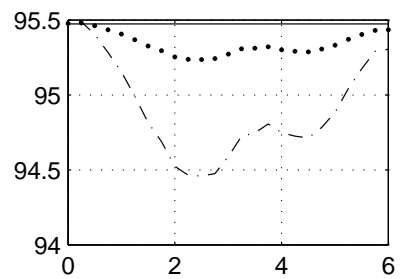

(c) III

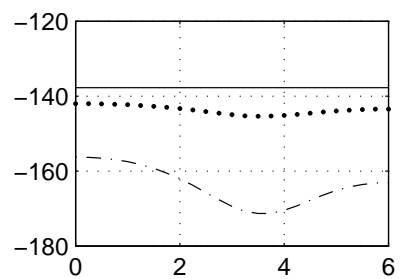

(f) $V I$

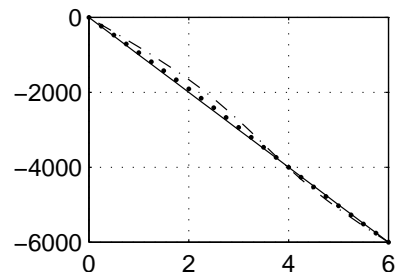

(i) $I X$

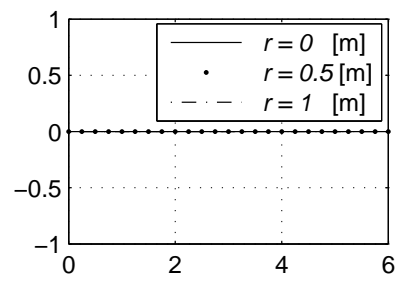

(l) XII

Figure 12: FEM simulation of the measurements $\left(u \times 10^{-5}[\mathrm{~mm}], w \times 10^{-5}[\mathrm{~mm}]\right.$ or $\left.\phi \times 10^{-2}[\mathrm{~V}]\right)$, for every position in the measuring boundary $(z[\mathrm{~cm}])$, for several sizes of the defect, and for all configurations in Table 1.

- CF functions with steep minima are considered to better than those providing soft valleys, due to algorithm convergence performance considerations.

- CF functions with oval valleys indicate that the parameters are coupled. In this case, the position of the minimum along the valley is difficult to find by the algorithms (although the location along the transverse direction is easy). For this reason, valleys with shapes close to circular are considered to be better.

Figure 13 shows the CF (7) as functions of the parameters $x_{0}$ and $z_{0}$, when the defect is located at $\tilde{\mathbf{p}}=(3.5,2,0.5)[\mathrm{cm}]$. Following the previously stated criteria, the best configurations are considered to be,

$$
C_{2}=\{I I, I I I, V, V I\}
$$


Furthermore, Figure 13 allows to extract two relevant conclusions: i) mechanical loads are better than electrical ones for exciting the system in damage evaluation, and ii) the optimal measurement magnitudes are the voltage $\phi$ and the vertical component of the displacement $w$.

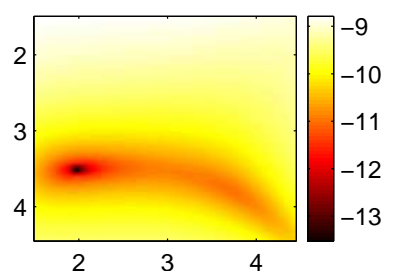

(a) $I$

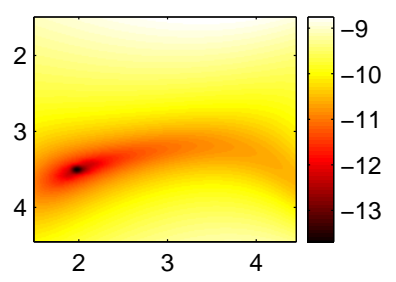

(d) $I V$

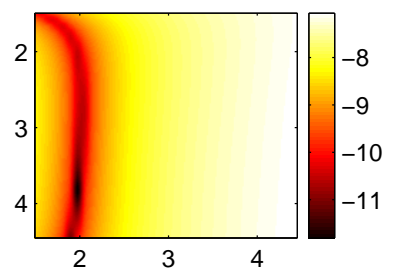

(g) VII

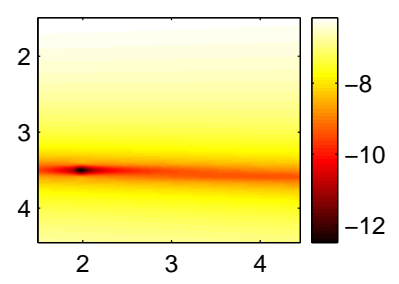

(j) $X$

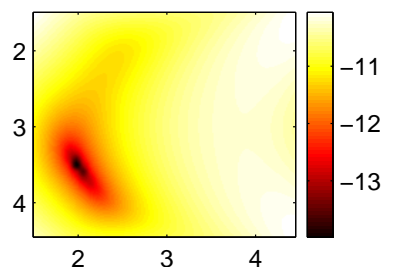

(b) $I I$

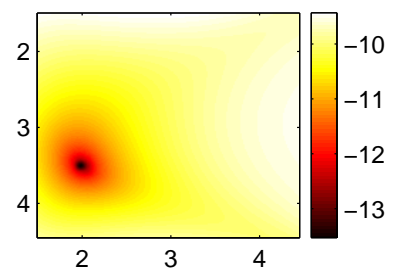

(e) $V$

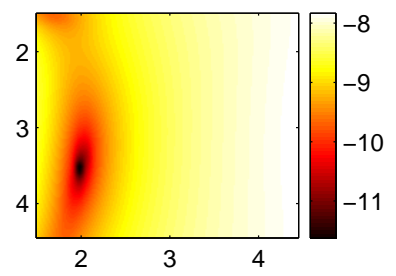

(h) VIII

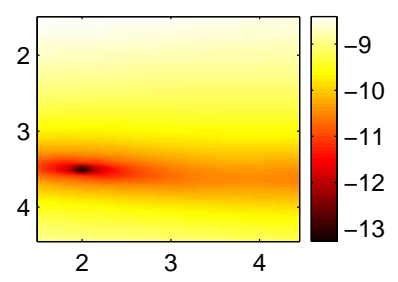

(k) $X I$

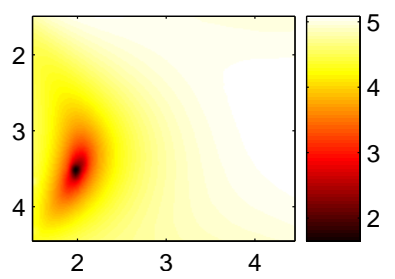

(c) $I I I$

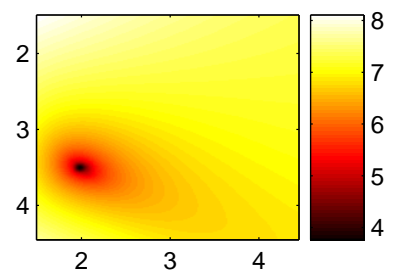

(f) $V I$

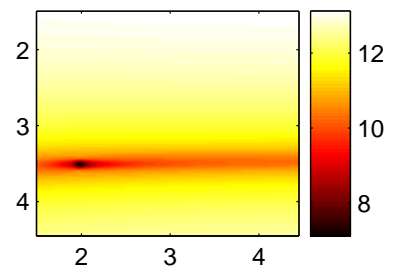

(i) $I X$

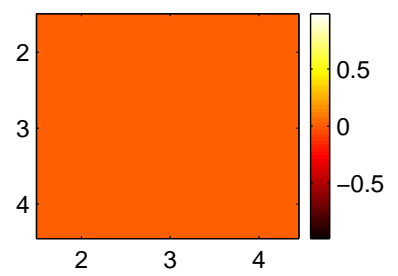

(1) $X I I$

Figure 13: Cost functional (in grayscale) as a function of the parameters $x_{0}[\mathrm{~cm}]$ and $z_{0}[\mathrm{~cm}]$ when the defect is located at $\tilde{\mathbf{p}}=(3.5,2 ., 0.5)[\mathrm{cm}]$.

Figure 14 shows the CF, computed in the same conditions as in Figure 13, but as a function of the parameters $z_{0}$ and $r$. In these plots, the best shapes of the $\mathrm{CF}$ are,

$$
C_{3}=\{I I, I I I\}
$$

\subsection{Effects on POD}

The criteria presented in the preceding sections is aimed at the local behaviour of the cost functional, regardless of the noise effects. A different criteria is here studied, which is to maximize the POD (i.e. 


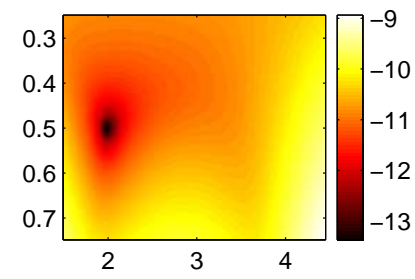

(a) $I$

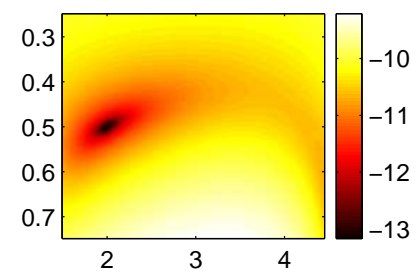

(d) $I V$

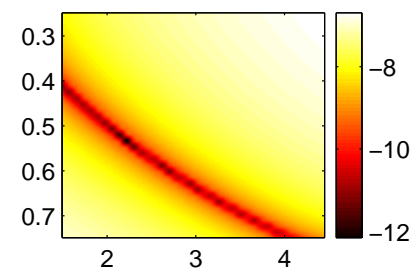

(g) VII

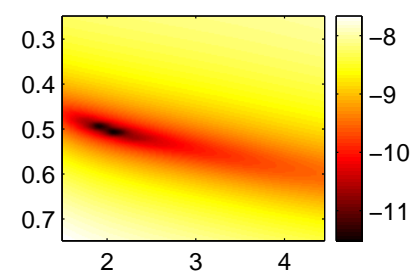

(j) $X$

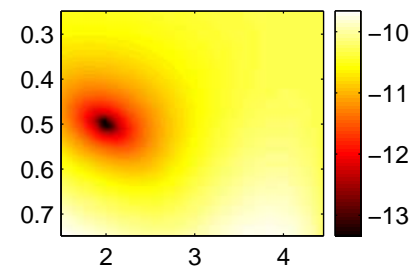

(b) $I I$

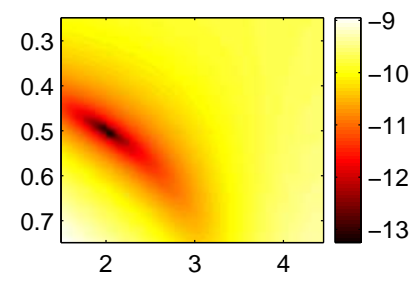

(e) $V$

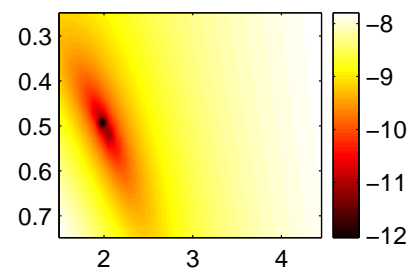

(h) VIII

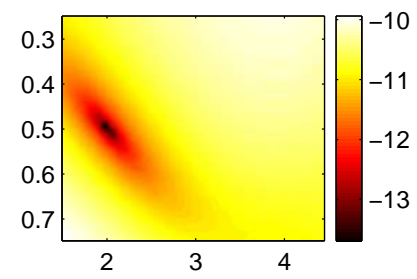

(k) $X I$

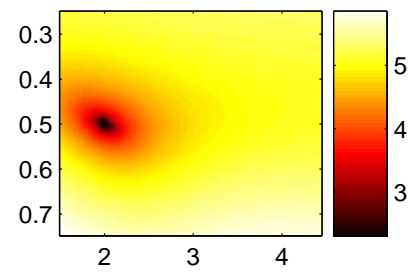

(c) $I I I$

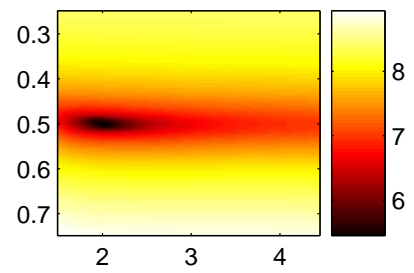

(f) $V I$

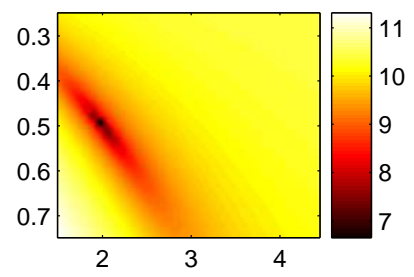

(i) $I X$

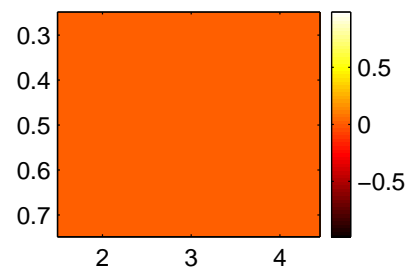

(1) $X I I$

Figure 14: Cost functional as a function of the parameters $r[\mathrm{~cm}]$ and $z_{0}[\mathrm{~cm}]$ when the defect is located at $\tilde{\mathbf{p}}=(3.5,2 ., 0.5)[\mathrm{cm}]$.

being able to find the smallest defect given the largest noise levels), independently of the robustness of the convergence of the search. For this reason, all three points of view provide conditioning information in order to select the most robust experimental setup.

In order to estimate the POD, since every possible location of the defect yields a different value of the POD, according to our formulation (a defect close to the sensors is usually easier to find than one far from them), a grid of possible defect locations is evaluated for each function, and the most pessimistic one is adopted. Figure 15 shows an example of the POD estimation for configuration III for all 16 tested locations of the defect. The left figure shows the dependency of the POD on the damage extent (area $A$ ), given a fixed and realistic noise level of $\sigma=2 \%$ (see [18]), whereas the right figure shows the dependency of the POD against the noise level, fixing the damage extent at $A=1\left[\mathrm{~cm}^{2}\right]$, which is a realistic damage threshold. The POD in all cases presents a similar trend with a narrow range of variation (around $20 \%$ in terms of area, or $40 \%$ of noise). As expected, the 
easiest defects to find are the ones closest to the sensors (position 4, against 16, which is the worst one, since asymmetrical boundary conditions were applied, see Figure 11). To give an idea of the extent of uncertainty in damage using this configuration, a value of $A=1.07\left[\mathrm{~cm}^{2}\right]$ is detectable at a standard noise of $2 \%$ with a probability of detection $P O D=99.9 \%$.
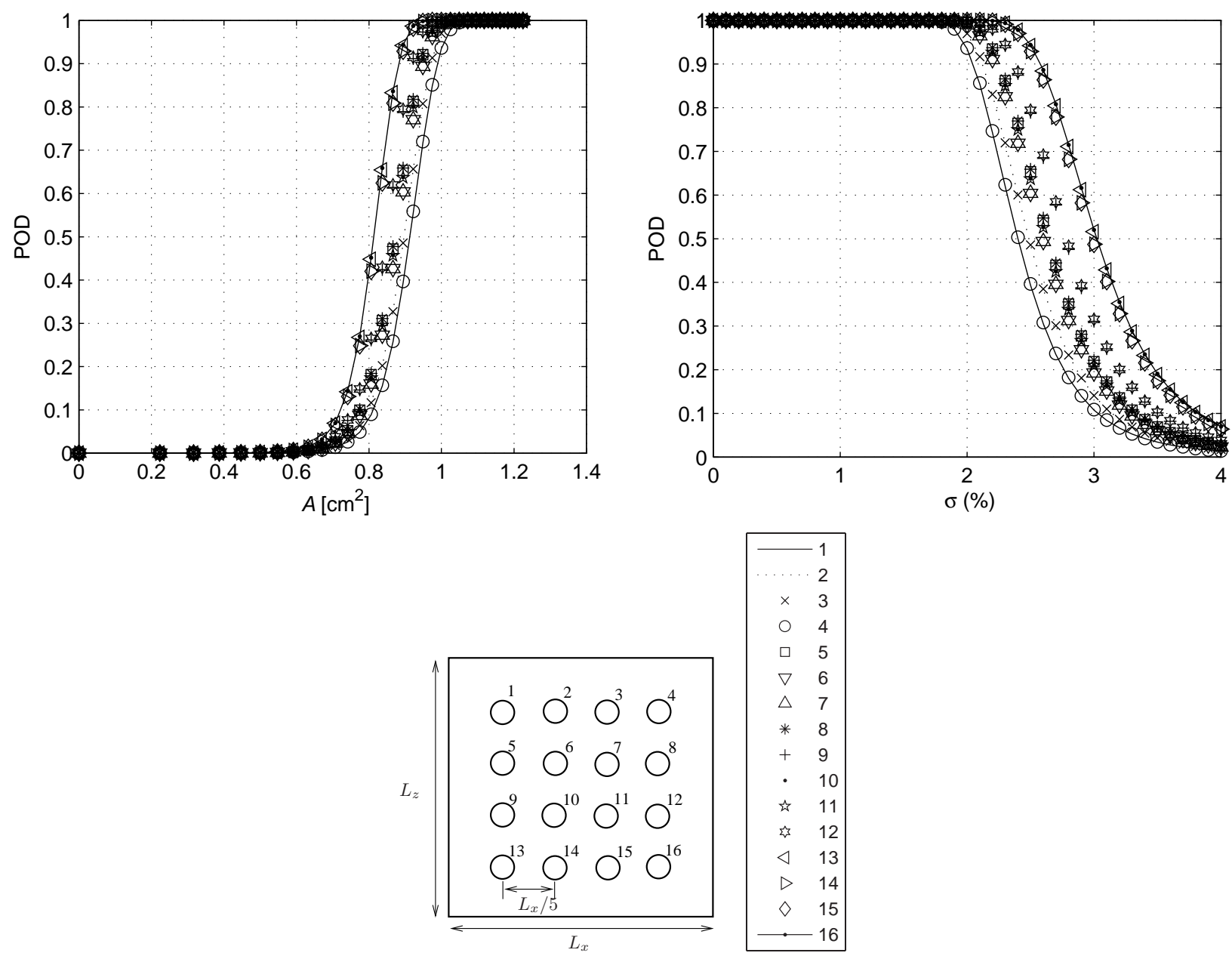

Figure 15: Dependency of the POD on the defect location in the plate.

Figure 16 shows the non-dimensional relative area of the defect $A /\left(L_{x} L_{z}\right)$ that can be found within a confidence interval of $95 \%$ and $99.9 \%$ as a function of the dimensions of a rectangular specimen $L_{x} / L_{z}$, using expression (16). In this case, the defect is always assumed at the center of the plate. A large sensitivity to the geometry of the plate is observed for all configurations except for $V I, V I I, I X, X I$ and $X I I$. Moreover, configurations $I, I I, I V$ and $V I I I$ provide the smallest findable defects and, hence, present the largest POD given a fixed size and noise level.

In order to establish a quantitative comparison between excitation-measuring configurations, the minimal and median numerical values of the Relative Area detectable to $\mathrm{POD}=99.9 \%$ is shown in Table 2 for every configuration. This table is derived from the Relative Area data from Figure 16. This figure concludes that the configuration which allows to find the smallest defect is IV (measuring displacement $u$ while exciting with stress in direction $z, T_{z z}^{a p}$ ). This conclusion is only based on one criteria, and should therefore just recommend a set of good configurations to be considered under 


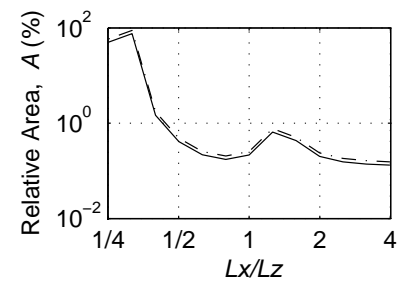

(a) $I$

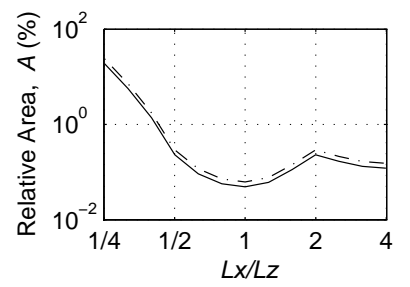

(d) $I V$

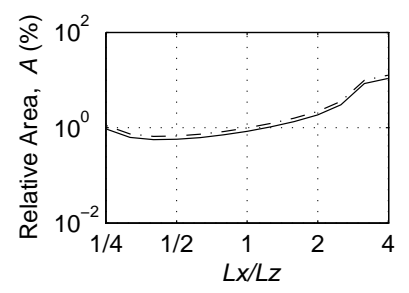

(g) $V I I$

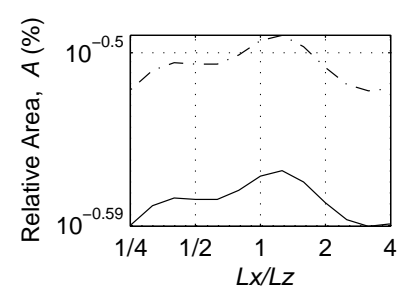

(j) $X$

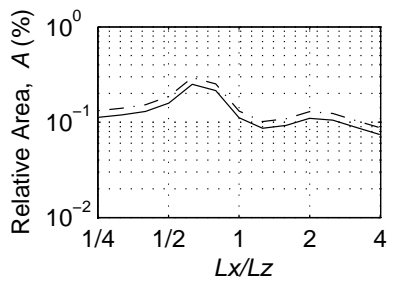

(b) $I I$

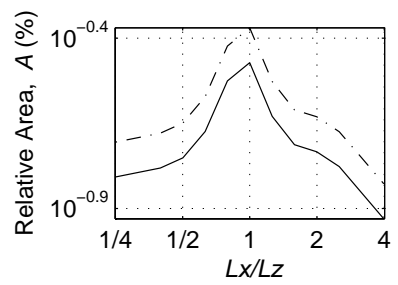

(e) $V$

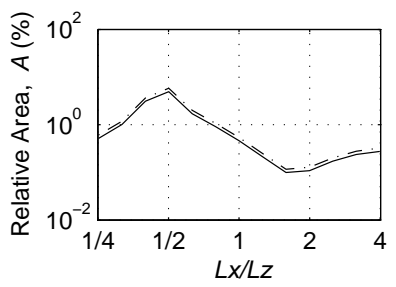

(h) VIII

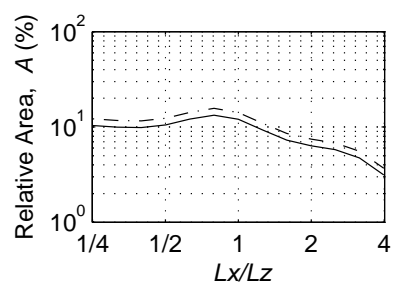

(k) $X I$

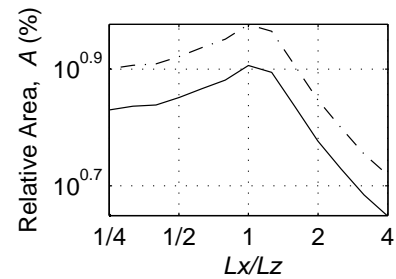

(c) $I I I$

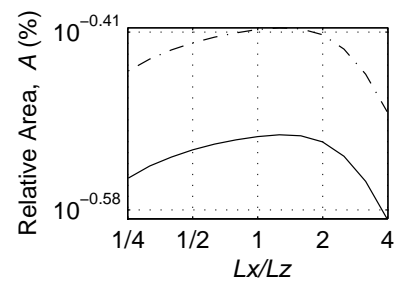

(f) $V I$

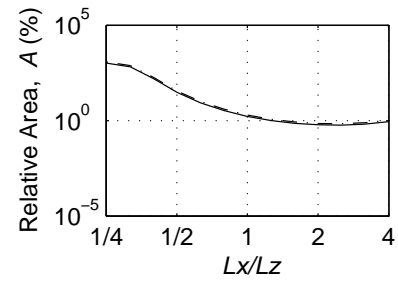

(i) $I X$

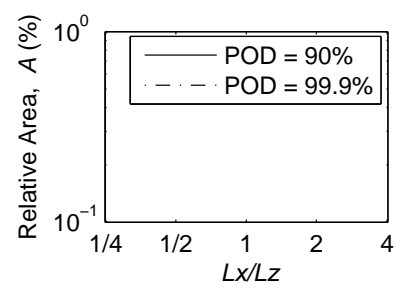

(l) $X I I$

Figure 16: Dependence of the size of the smallest findable defect on the shape of the plate and on the measurement/loading combination.

additional criteria.

Independently of this, the detection works better while measuring displacements, since these magnitudes are shown to be more sensitive to the defect. However, configuration III, whose minimum is easier to find according to the CF criteria, finds defects in the less favourable case of $\approx 3\left[\mathrm{~m}^{2}\right]$ in a $36\left[\mathrm{~m}^{2}\right]$ plate. In addition, the longer the $L_{z}$ side is with respect to $L_{x}$, the easier it is to find a defect.

In general, the largest POD seems to be achieved when the direction of polarization is the same as the longest side of the plate (in this case $L_{z}>>L_{x}$ ). 


\begin{tabular}{|c|c|c|c|}
\hline Load $\quad$ Measurement & $u$ & $w$ & $\phi$ \\
\hline$T_{x x}^{a p}$ & 0.15 & 0.09 & 9.48 \\
\hline$T_{z z}^{a p}$ & $\begin{array}{ll}0.05 & 0.05 \\
\end{array}$ & 0.12 & 0.32 \\
\hline$E_{x}^{a p}$ & $\begin{array}{ll} & 0.66\end{array}$ & $\begin{array}{ll}0.54 & 0.12 \\
\end{array}$ & 1.98 \\
\hline$E_{z}^{a p}$ & $\begin{array}{ll}0.32 & 0.30\end{array}$ & $14.14 \quad 3.68$ & \\
\hline
\end{tabular}

Table 2: Relative Area $A$ detectable to $\mathrm{POD}=99.9 \%$ for different excitation-measuring configurations. The two figures in each box correspond to the minimal (left) and median (right) cases respectively.

\subsection{Optimal configuration}

Following the criteria and the conclusions from the former sections, the best configurations to find the minimum may be considered as the intersection of the three sets $C_{1}, C_{2}$ and $C_{3}$,

$$
C_{1} \cap C_{2} \cap C_{3}=\{\mathrm{II}, \mathrm{III}\}
$$

Nevertheless, from an experimental viewpoint, it is easier to measure voltages rather than displacements, in addition to more accurate. For this reason, we may conclude that the optimal configuration is III (voltage $\phi$ with applied stress in direction $x, T_{x x}^{a p}$ ). Furthermore, as observed in Figure 12, this configuration provides measurements with high sensitivity to the size of the defect.

\section{Inverse problem solution}

In this section, the results of minimizing the CF by GA and BFGS are compared, and the effect of the noise on the output parameters is investigated.

Figure 17 shows the CF of configuration III ( $\phi$ under $T_{x x}^{a p}$ applied) when increasing levels of simulated experimental noise are considered: $\sigma=0.01,0.05,1,2 \%$. As expected intuitively, the minimum is observed to become less accessible as the noise applied on the measurements $\phi^{E X P}$ is increased.

In the next sections, defect searches (CF optimizations) are performed using GA and BFGS (for the case of configuration III and for increasing levels of noise).

\subsection{Defect search using GA}

In order to perform the GA optimization, the selected population size should guarantee to find a global optimum at an adequate computational cost. This saving is due to the selection of the configuration optimization performed in the previous section. Despite this, genetic diversity has to be injected to the mutation and crossover parameters in order to ensure that the solution does not fall in local minima. Finally, Table 3 shows the parameters used. 

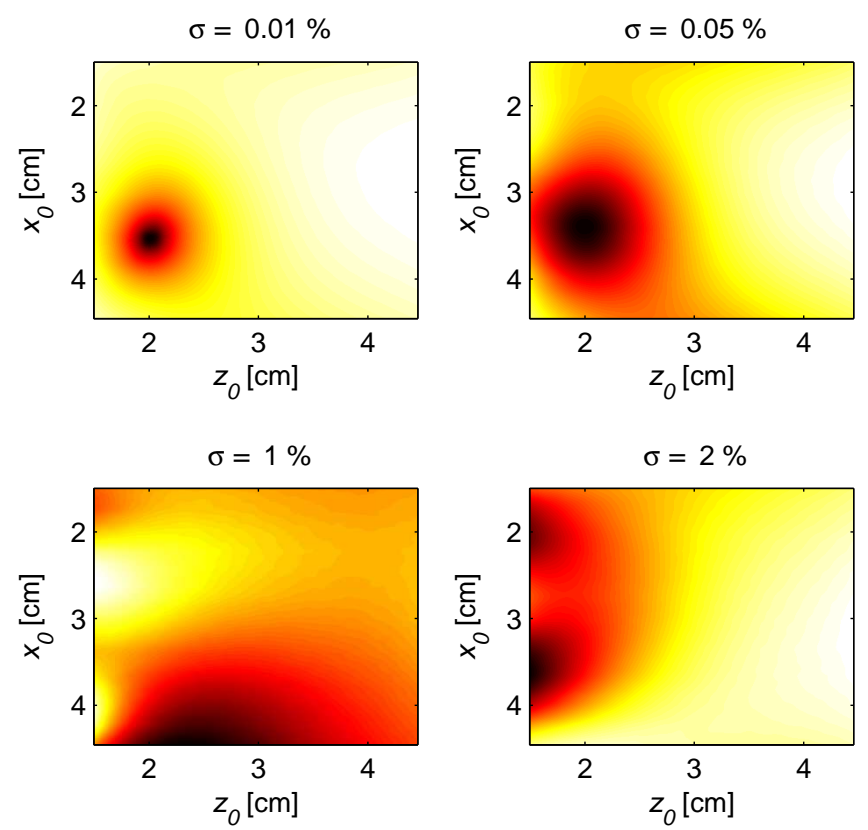

Figure 17: Cost functional for increasing levels of experimental noise.

\begin{tabular}{||c|c||}
\hline Parameter & Value \\
\hline \hline Population size $(\mathrm{Ps})$ & 50 \\
\hline Crossover ratio $(\mathrm{Cr})$ & 0.9 \\
\hline Mutation ratio $(\mathrm{Mr})$ & 0.2 \\
\hline Number of generations $(\mathrm{Ng})$ & 150 \\
\hline
\end{tabular}

Table 3: Parameters used for the GA minimization.

Figure 18 shows the CF (7) as a function of the generation number in the GA minimization procedure, for the case without noise. The FC oscillates in the range $12-6$ until generation 125 , and then a rapid convergence is observed towards approximately 2, which provides parameters coinciding with the minimum seen on Figure 13 and 14 for configuration III.

\subsection{Defect search by BFGS}

The BFGS search was performed 100 times with a different random initial guess each time. $39 \%$ of these searches converged successfully (the criteria for convergence is set as arriving to a solution within 0.01 distance, see (17), from the real one. Figure 19(left) shows the distances of the converged solutions versus the distance of the initial guess to the real defect. The dependency of the convergence on the initial guess is shown since the converged results (dots) are concentrated at low initial distances.

Figure 19(right) presents the evolution of the $\mathrm{CF}$ and the three parameters that define the defect during the iterations of a successful search procedure (starting from the initial guess of $(x=3.9290, y=2.6270, r=0.3406)[\mathrm{cm}]$, which provides the optimum result among the tested random initial positions). It should be noted that the cost functional used for BFGS is $f$ as defined 


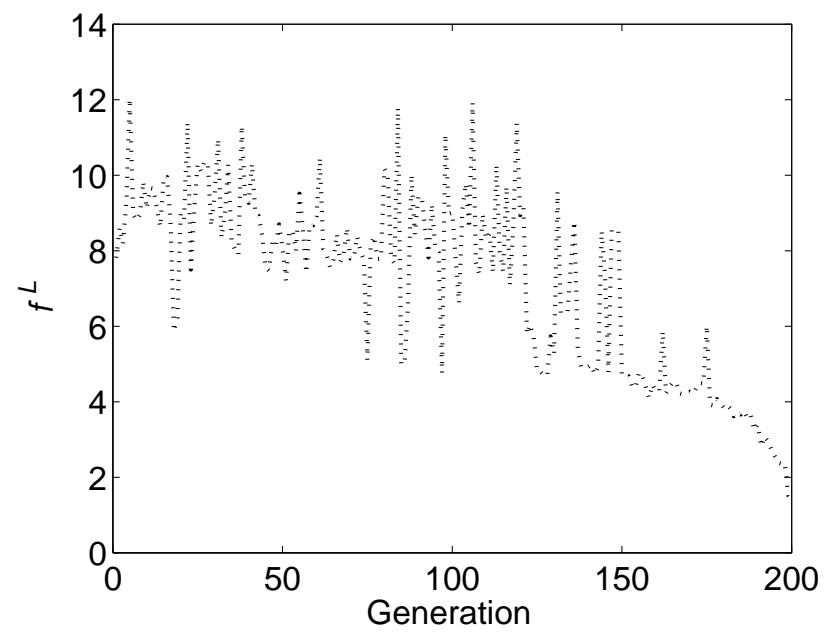

Figure 18: CF for every generation in the GA minimization.

in (6), whereas the CF used with GA is $f^{L}$ defined in (7).

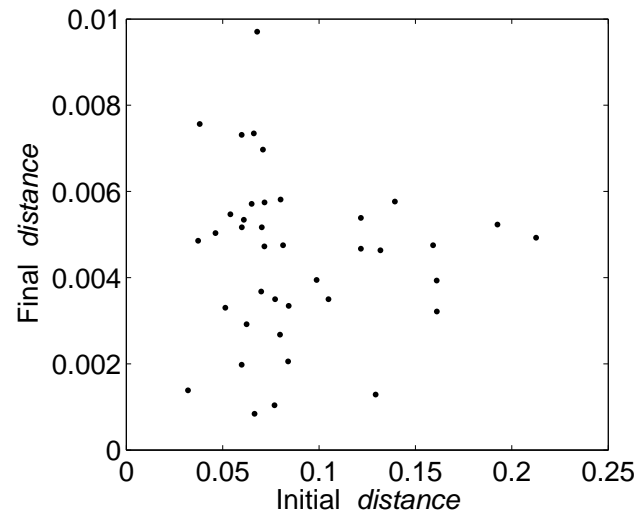

(a)

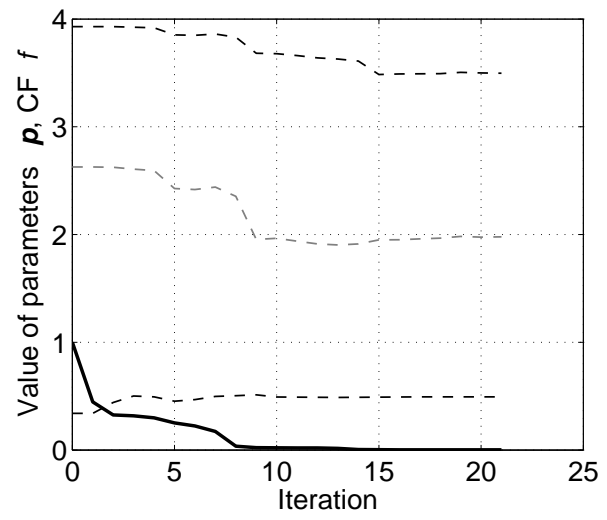

(b)

Figure 19: Distance of the converged searches using BFGS (left) and convergence process for a single search (right). The $\mathrm{CF}$ is represented by the thick line, which converges to zero, and the three parameters (initially $(x=3.9290, y=2.6270, r=0.3406)[\mathrm{cm}])$ with discontinuous line.

\subsection{GA compared to BFGS}

In order to formalize a comparison between GA and BFGS convergence for different levels of noise, a distance between predicted and real parameters is defined in an Euclidean sense as:

$$
\text { distance }=\frac{\sqrt{\sum_{i=1}^{N}\left(\tilde{p}_{i}-p_{i}^{c}\right)^{2}}}{\sum_{i=1}^{N} \tilde{p}_{i}}
$$

where $N$ is the number of parameters to identify. 
Figure 20 shows the distance versus the simulated measurement noise. It is observed how this measurement of distance or badness of the output increases with noise for both optimization methods. From this figure, some advantages may be concluded for the BFGS and GA methods respectively.

The BFGS method is more reliable than GA for noise levels below 2\%, since it converges to lower distances. This algorithm converges to more precise solutions especially for moderate noise levels. However, in [18] was concluded that the noise level in experimental cases should be of the order of magnitude of $2 \%$, which reduces the aforementioned advantage. On the other hand, the BFGS algorithm is more operative from a computational point of view, since it requires fewer $\mathrm{CF}$ evaluations and therefore FEM computations. In particular, the GA solution requires 6032 FEM simulations, whereas the BFGS solution for the specified initial point requires 108 FEM simulations.

The GA procedure guarantees convergence, most probably to the global minimum (if used correctly), whereas the convergence of the BFGS algorithm strongly depends on the initial guess that needs to be provided. The probability of convergence was shown to be $39 \%$ in the previous section, and increases with the goodness of the initial guess. For this reason, GA is preferred as long as the computational cost is affordable.

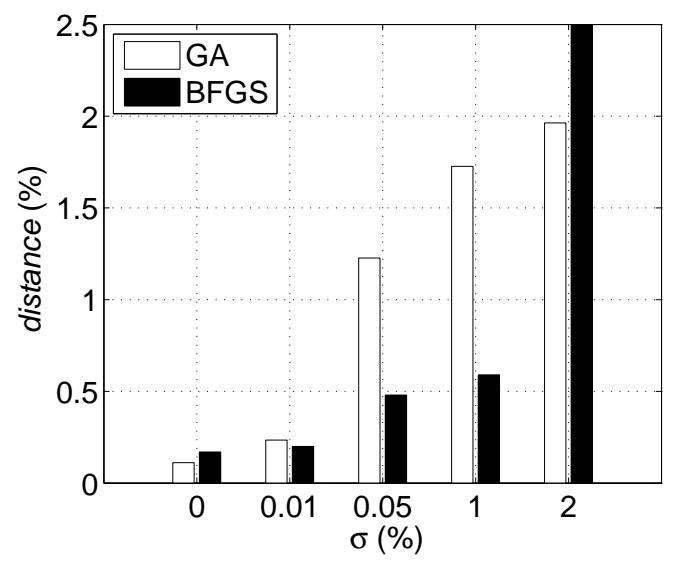

Figure 20: Distance between computed and real results as a function of noise level.

Finally, Figure 20 suggests that using the procedure developed in this work, it is possible to find a defect with an error smaller than 5 times the error on the measurement.

\section{Conclusion}

A numerical method to determine the location and extent of defects in piezoelectric plates is developed by combining the solution of an identification inverse problem, using genetic algorithms to minimize a cost functional, and using an optimized finite element code and meshing algorithm.

In addition, an analytical estimate of the probability of detection is developed and validated with a Monte Carlo simulation for the first time. This allows to estimate a priori the minimum defect findable given a specimen geometry and a noise level on measurements.

These tools are used to numerically study and compare different excitation and measuring setups and geometries for evaluating the damage. This study concludes that the measurement of voltage 
$\phi$ provides the highest sensitivity to the defect and lowest to noise (and therefore identifiability), combined with an excitation of the specimen by a horizontal traction $T_{x x}^{a p}$, i.e. transversely to the polarization of the piezoelectric ceramic. Just to give some quantitative figures, it is concluded that the presented technique would allow to successfully and accurately locating and sizing a defect from measurements with realistic levels of noise (more than 1\%).

Two search procedures are compared, based on Genetic Algorithms and the BFGS algorithm. The first one showed a more robust convergence as long as its higher computational cost is affordable, whereas the second may provide more precise results for moderate noise levels in measurements.

To give an idea of the extent of uncertainty in damage detection using the proposed technique and experimental setup, a damage extent of $A=1.07\left[\mathrm{~cm}^{2}\right]$ is detectable at a standard noise of $2 \%$ with a probability of detection $P O D=99.9 \%$.

\section{Acknowledgment}

This research was supported by the Ministry of Education of Spain through grant number FPU AP-2006-02372.

\section{References}

[1] Araújo AL, Mota Soares CM, Herskovits J and Pedersen P. Development of a finite element model for the identification of mechanical and piezoelectric properties through gradient optimisation and experimental vibration data. Composite Structures 2002; 58(5): 307-318.

[2] Araújo AL, Mota Soares CM, Herskovits J and Pedersen P. Parameter estimation in active plate structures using gradient optimisation and neural networks. Inverse Prob. in Sci. and Eng. 2006; 14(5): 483-493.

[3] Benjeddou A. Advances in piezoelectric finite element modeling of adaptive structural elements: a survey. Computers and Structures 2006; 76: 347-363.

[4] Bonnet $\mathrm{M}$ and Constantinescu A. Inverse problems in elasticity. Inverse Problems 2005; 21: R1-R50.

[5] Chou JH and Ghaboussi J. Genetic algorithm in structural damage detection. Comput. Strut. 2001; 79: 1335-1353.

[6] Dennis JE and Schnabel RB. Numerical Methods for Unconstrained Optimization and Nonlinear Equations. SIAM, 1983.

[7] Gallego R and Rus G. Identification of cracks and cavities using the topological sensitivity boundary integral equation. Comp. Mechanics 2004; 33: 154-163.

[8] Goldberg DE. Genetic Algorithms in search, Optimization and Machine Learning. AddisonWesley, 1989.

[9] Jelitto H, Kessler H, Schneider GA and Balke H. Fracture behavior of poled piezoelectric PZT under mechanical and electrical loads. J. of European Ceramic Society 2005; 25: 749-747. 
[10] Kaltenbacher B, Lahmer T, Mohr M and Kaltenbacher M. PDE based determination of piezoelectric material tensors. Europ. J. App. Mathem. 2006; 17: 383-416.

[11] Kuna M. Finite element analyses of cracks in piezoelectric structures: a survey. Arch. Appl. Mech. 2006; 76: 725-745.

[12] Liu PL and Chen CC. Parametric identification of truss structures by using transient response. J. Sound Vib. 1996; 191: 273-287.

[13] Mares C and Surace C. An application of genetic algorithms to identify damage in elastic structures. J. Sound Vib. 1996; 195: 195-215.

[14] McMeeking RM. The energy release rate for a Griffith crack in a piezoelectric material. Eng. Fract. Mech. 2004; 71: 1149-1163.

[15] Oh J, Cho M and Kim JS. Dynamic analysis of composite plate with multiple delaminations based on higher-order zigzag theory. Int. J. Solids 6 Struct. 2005; 42: 6122-6140.

[16] Ou ZC and Chen YH. Discussion of the crack face electric boundary condition in piezoelectric fracture mechanics. Int. J. of Fracture 2003; 123: L151-L155.

[17] Pagano NJ, Exact solution for rectangular bidirectional composites and sandwich plate. J. Comp. Mater. 1970; 4: 20-34.

[18] Palma R, Rus G and Pérez-Aparicio JL. Sensitivity of inverse problem solution to system uncertainties for piezoelectric ceramics submitted 2007.

[19] Park S and Sun CT. Fracture criteria for piezoelectric ceramics. J. Amer. Ceram. soc. 1995; 78: $1475-1480$.

[20] Pérez-Aparicio JL, Sosa H and Palma R. Numerical investigations of field-defect interactions in piezoelectric ceramics. Int. J. Solids \& Struct. 2007; 44: 4892-4908.

[21] Råde L, Westergren B. Mathematics Handbook for Science and Engineering. Springer, 1999.

[22] Ruíz A, Ramos A and San-Emeterio JL. Evaluation of piezoelectric resonator parameters using an artificial intelligence technique. Integrated Ferroelectric 2004; 63: 137-141.

[23] Ruíz A, Ramos A and San-Emeterio JL. Estimation of some transducer parameters in a broadband piezoelectric transmitter by using an artificial intelligence technique. Ultrasonics 2004; 42: 459-463.

[24] Rus G, Lee S and Gallego R. Defect identification in laminated composite structures by BEM from incomplete static data. Int. J. Solids \& Struct. 2005; 42: 1743-1758.

[25] Rus G, Lee S Y, Chang SY and Wooh SC. Optimized damage detection of steel plates from noisy impact test. Int. J. Numer. Meth. Engng. 2006; 68(7): 707-727.

[26] Russell RD and Ghomshei MM. Inverting piezoelectric measurements. Tectonophysics 1997; 271: 21-35. 
[27] Saltelli A, Chan K and Scott EM. Sensitivity Analysis. John Wiley \& Sons LTD, 2000.

[28] Sosa H and Khutoryansky N. New developments concerning piezoelectric materials with defects. Int. J. Solids \& Struct. 1996; 33(23): 3399-3414.

[29] Suo Z, Kuo CM, Barnett DM and Willis JR. Fracture mechanics for piezoelectric ceramics. J. Mech. and Phy. of Solids 1992; 40: 739-765.

[30] Tam TKH and Armstrong CG. 2D Finite element mesh generation by medial axis subdivision. Adv. Eng. Software 1991; 13.

[31] Tarantola A and Valette B. Inverse problems = quest for information. J. Geophys. 1982; 50: $159-170$.

[32] Tardieu N and Constantinescu A. On the determination of elastic coefficients from indentation experiments. Inverse Problems 2000; 16: 577-588.

[33] Taylor RL 2005 F.E.A.P.- A finite analysis program. User manual U of California, Berkeley www.ce.berkeley.edu/ ${ }^{\sim}$ rlt

[34] Thompson JF, Soni BK and Weqtherill NP. Handbook of Grid Generation. CRC London, 2003.

[35] Vatul'yan AO, Dombrova OB and Zhirov VY. Inverse problems for non-uniformly polarized piezoelectric rods. J. App. Mathem. and Mechanics 2007; 71: 85-92. 